\title{
Renal involvement in children with COVID-19 infection
}

\author{
Hossein Emad Momtaz* \\ Division of Pediatric Nephrology, Hamadan University of Medical Sciences, Hamadan, Iran
}

\section{A R T I C L E I N F O}

Article Type:

Mini Review

\section{Article History:}

Received: 20 April 2020

Accepted: 30 April 2020

Published online: 9 May 2020

\section{Keywords:}

COVID-19

Children

Kidney

Acute kidney injury

Coronavirus

\begin{abstract}
A B S T R A C T
Perhaps when China reported its first cases of the novel coronavirus in December 2019, few would predict that it would overwhelm the majority of the global community. The first reports conveyed that the rate of infection and death from this virus among children is rare. However, evidence showed that there is no particular age range for the disease and children, infants and even neonates may be infected. Although COVID-19 primarily targets the host's respiratory system, complications in other organs such as heart, kidney and liver have been observed as well. This mini-review attempts to consider the publications focused on the COVID-19 infection among children with emphasis on renal involvement and the treatment approach of this complication.
\end{abstract}

Implication for health policy/practice/research/medical education:

Initially, COVID-19 infection was supposed to rarely involve pediatrics, now it is evident that even newborns may be infected. Kidneys may be of important target organs of the virus due to direct invasion or inflammation.

Please cite this paper as: Momtaz HE. Renal involvement in children with COVID-19 infection. J Renal Inj Prev. 2020; 9(3): e20. doi: 10.34172/jrip.2020.20.

\section{Introduction}

Coronavirus belongs to the RNA virus family which is able to infect both humans and animals. Other strains of this virus were known to be responsible for human upper respiratory infections, which present themselves with symptoms similar to the common cold such as fever, runny nose and sore throat, self-resolving in a few days without creating serious complications or requiring significant treatment. However, in the past 18 years ago, two strains of the coronavirus family began to exhibit different behavior.

First, in 2003, cases of the severe acute respiratory syndrome (SARS) were reported and then in 2012, the Middle East respiratory syndrome (MERS) involved various countries, both caused by strains of coronavirus. The novel coronavirus or COVID-19, however, reported first in the Chinese city of Wuhan on December 31, 2019 has burdened the majority of the global community and has left tens of thousands of people dead.

SARS, which first cases of this disease were reported in
November 2002 in China, infected 8098 people from 29 countries resulting in 774 deaths. SARS was characterized to infect fewer children than adults and showed milder symptoms as well as quicker recovery in pediatric age group. In a study on patients clinically suspected to SARS, only 135 cases aged below 18 years were reported, among whom only 80 cases were confirmed by laboratory tests while there was no mortality among them.

First cases of MERS were reported in Saudi Arabia in September 2012 and infected 701 people by June 2014, while 249 of them died. Like SARS, MERS mostly infected adults and only 14 of the patients (2\%) were children while 2 of them eventually lost their lives.

As the recent coronavirus infection (COVID-19) caused an unprecedented pandemic in the world, healthcare professionals suggested that the probability of infection and mortality among children will be extremely low based on epidemiological estimates derived from experiences with MERS and SARS infections. Yet, more recent reports began to show different patterns of involvement in 
children like renal disturbance.

Methods and Materials

In order to undertake this review, the keywords "COVID-19", "Children", "Kidney", "Proteinuria", "Hematuria" and "acute kidney injury" were searched in PubMed, EMBASE, Cochrane and Scopus databases, implementing the most relevant publications.

\section{Results}

According to an article published in March 2020 by Qiu et al, in 36 children under the age of 16 years with confirmed COVID-19 infection, the most common route of transmission in $89 \%$ of children was close contact with infected family members. The average age of infected children was 3.8 years old. Around $47 \%$ of patients had mild symptoms while $28 \%$ were asymptomatic and $19 \%$ showed acute respiratory symptoms.

The most common symptoms were fever and dry cough and the most common laboratory findings were an increase in procalcitonin, a high CPK-MB and lymphopenia. None of the patients showed an increase in plasma urea and creatinine since all were successfully treated with medical treatment (1).

In a study by Sun et al in March 2020, findings of 8 children from 2 months to 15 years old infected by COVID-19 were analyzed. The most common symptoms included tachypnea, fever and coughs respectively. Chest $\mathrm{X}$-ray in 7 patients showed multiple patchy involvements with ground glass appearance in 6 patients. Leukocyte counts in 7 patients were high and in 6 patients an increase of C-reactive protein (CRP), lactate dehydrogenase (LDH) and procalcitonin was detected. Two patients required ventilators. Only two patients experienced an increase in serum creatinine but none required dialysis. Finally, five patients recovered and were discharged while three remained in pediatric intensive care unit (PICU) (2).

In a report by the Korean center for infectious disease research (KCDC), of 4212 confirmed COVID-19 cases, 201 were children, 32 of which were below 9 years of age and the other 169 were between 10 to 19 years old. The youngest patient was 45 days old and the majority of children experienced mild symptoms with no deaths reported (3).

Similarly, Zheng et al studied 25 COVID-19 infected children. The average age was three years old since $40 \%$ was below 3 years. The most common symptoms were fever and dry coughs. Chest X-ray was normal in 8 patients (3.33\%), however unilateral lung involvement in 5 patients and bilateral involvement in 11 patients was seen. Two patients with severe infection required ventilators and the same patients experienced an increase in serum creatinine, while one of them was treated with continuous venovenous hemodiafiltration (CVVHDF) and plasmapheresis resulting in significant improvement.
No deaths were reported among the children (4).

Ji et al reported two children with 9 and 15 years of age who were positive for COVID-19. They had the history of the COVID-19 infection in close family contacts. Both patients showed mild respiratory and gastrointestinal symptoms, and their chest X-ray results were normal with no involvement in other organs. They completely recovered through supportive treatments (5). Additionally, Su et al compared 5 children infected with COVID-19 to 14 infected adults from their family and concluded that children commonly contract the infection from family members. Although the symptoms are milder and prognosis is better, fecal excretion of virus continues longer than adults. Due to mild symptoms or asymptomatic cases, diagnosis proves to be more difficult. In their report, renal function of all the children was normal and no deaths were reported (6).

Accordingly, Dong et al in a study reported that among 2143 children presumed to be infected with COVID-19, 731 were tested positive while 1412 were suspicious of infection. Most of them showed mild symptoms and only $5.9 \%$ suffered from the severe form of the illness. This is significantly lower than the rate of severe cases in adults that were reported to be $18.5 \%$. Only one death, in a 14-year-old boy, was reported in this study (7).

In a recent study among 72314 cases of COVID-19 in China, Lu et al detected only $1 \%$ of COVID-19 in children below 10 years old. The average age of the infected children was 6.7 years since $15.8 \%$ showed no clinical findings or imaging findings in favor of infection. In $7 \%$ of infected children; despite radiological findings, no clinical symptoms were observed. Among these patients only 3 required PICU, while all of them with associated conditions such as hydronephrosis, leukemia and intussusceptions. Only one death, in a 10-month-old infant with intussusception and multiple organ failure, was reported (8).

Likewise, the recent study by Naicker et al in March 2020 suggested that COVID-19 infection can be seen in all ages including children, with children experiencing rhinorrhea and productive cough more than adults. However, severe symptoms were not common among children (9).

\section{Kidney involvement in COVID-19}

While in previous infections such as SARS and MERS, acute kidney injury (AKI) was reported to be up to $15 \%$ with a mortality rate of approximately $60 \%$ to $90 \%$, however initial reports of COVID-19 suggest a lower rate in adults (3\% to $9 \%)$.

In the study by Wang et al, out of 138 adults infected with COVID-19, five (6.3\%) suffered from AKI. There was no significant difference of AKI prevalence in patients admitted in ICU and the patients admitted in other wards(10).

Moreover, Li et al observed that in infected patients by 
COVID-19, urinalysis showed proteinuria and hematuria. This finding was observed in both severe and mild cases without significant difference. Additionally, 31\% of the patients had raised BUN and $22 \%$ showed an increase in serum creatinine. Among these patients 7 patients (4\%) required continuous renal replacement therapy (CRRT), all of whom suffered from the severe form of the infection (11). More recently, in a study by Cheng et al on 710 adults infected with COVID-19, 44\% had proteinuria and hematuria. Additionally, $15.5 \%$ of the patients had raised serum creatinine and in $16.1 \%$ an increase in BUN was detected (12).

\section{Mechanism of kidney injury}

Yet, it is possible that the cause of renal impairment lays in the direct invasion of virus to the renal tubular cells or due to cytokine storm. Angiotensin-converting enzyme (ACE) and dipeptidyl peptidase-4 (DPP4) which reside on renal tubular cells were previously known as receptors for SARS and "human coronavirus Erasmus Medical Center" (hCOV-EMC ) viruses since in both of these infections the RNA of virus was extracted from renal tissues $(13,14)$. Recently COVID-19 was extracted from the patients' urine which may be an indicator for kidney involvement (15).

More recently, Pan et al demonstrated that the COVID-19 virus can cause direct injury to podocytes and proximal tubular cells through attaching to ACE2 receptors and cellular transmembrane serine protease enzyme activity which may explain proteinuria in COVID-19 patients. They also proposed that the simultaneous expression of ACE 2 receptor genes and cellular transmembrane serine protease in kidney cells are not less than in other organs, therefore kidney is also a target organ for the COVID-19 virus (16).

\section{Treatment}

Although no definitive treatment has been found at the time of this writing, several medications such as chloroquine phosphate, lopinavir/ritonavir, remdesivir, interferon alpha, favipiravir, umifenovir and tocilizumab have been suggested as likely effective medications in controlling the disease. There are several ongoing clinical trials regarding their effectiveness and side effects (17).

Steroids seem to increase mortality rate, according to previous studies conducted on MERS and SARS patients, since administration of these group of drugs is controversial and generally not recommended (18).

Use of recovered patients' plasma has shown promising results in primary studies but still requires further researches (19).

\section{COVID-19 and dialysis patients}

This infection can be a potential threat to dialysis patients. In a review, $16 \%$ of patients and $12 \%$ of staff in a dialysis ward were infected with COVID-19 since out of 7 expired patients, 6 were suffering from COVID-19 infection. Despite this risk, dialysis patients in general showed milder clinical symptoms, fewer cases of lymphopenia and lower levels of cytokines (20).

The use of CRRT in the treatment of severe COVID-19 cases has shown in some studies to be effective, however, more extensive researches are required to confirm this result (21).

\section{Conclusion}

Despite initial reports and background experiences of SARS and MERS cases which infection among children is rare, recent reports showed that all ages, even infants and neonates can be infected with the virus. Renal complications are reported to be limited to the severe cases in children, and are mostly presents as AKI in the context of multi-organ failure caused by direct virus invasion or acute inflammatory response. In a limited number of studies conducted among adults, proteinuria and hematuria were observed in patients. However, studies on children have not yet been conducted. More studies of renal complications in children with COVID-19 infection are recommended in initial stages of this disease.

Author's contribution

HEM searched the data, prepared, edited and finalized the paper. The author read and signed the final manuscript.

\section{Conflicts of interest}

The author declares that he has no competing interests.

\section{Ethical considerations}

Ethical issues (including plagiarism, data fabrication, double publication) have been completely observed by the author.

\section{Funding/Support}

None.

\section{References}

1. Qiu H, Wu J, Hong L, Luo Y, Song Q, Chen D. Clinical and epidemiological features of 36 children with corona virus disease 2019 (COVID-19) in Zhejiang, China: an observational cohort study. Lancet Infect Dis, 2020. doi: 10.1016/S1473-3099(20)30198-5

2. Sun D, Li H, Lu XX, Xiao H, Ren J, Zhang FR, et al. Clinical features of severe pediatric patients with corona virus disease 2019 in Wuhan: a single center's observational study. World J Pediatr. 2020 Mar 19. doi: 10.1007/s12519020-00354-4

3. Korean Society of Infectious Diseases; Korean Society of Pediatric Infectious Diseases; Korean Society of Epidemiology; Korean Society for Antimicrobial Therapy; Korean Society for Healthcare-associated Infection Control and Prevention; Korea Centers for Disease Control and 
Prevention Report on the Epidemiological Features of Coronavirus Disease 2019 (COVID-19) Outbreak in the Republic of Korea from January 19 to March 2, 2020. J Korean Med Sci. 2020;35:e112. doi: 10.3346/jkms.2020.35. e112

4. Zheng F, Liao C, Fan QH, Chen HB, Zhao XG, Xie ZG, et al. Clinical characteristics of children with corona virus disease 2019 in Hubei, China. Curr Med Sci. 2020. doi: 10.1007/s11596-020-2172-6

5. Ji LN, Chao S, Wang YJ, Li XJ, Mu XD, Lin MG, et al.Clinical features of pediatric patients with COVID-19: a report of two family cluster cases. World J Pediatr. 2020 Mar 16. doi: 10.1007/s12519-020-00356-2

6. Du W, Yu J, Wang H, Zhang X, Zhang S, Li Q, Zhang Z. Clinical characteristics of COVID-19 in children compared with adults in Shandong Province, China. Infection. 2020 Apr 16. doi: 10.1007/s15010-020-01427-2

7. Dong Y, Mo X, Hu Y, Qi X, Jiang F, Jiang Z, et al. Epidemiological characteristics of 2143 pediatric patients with 2019 coronavirus disease in China. Pediatrics. 2020 Mar 1. doi: 10.1542/peds.2020-0702.

8. Lu X, Zhang L, Du H, Zhang J, Li YY, Qu J, et al. SARSCoV-2 Infection in Children. N Engl J Med. 2020 Apr 23;382:1663-5. doi: 10.1056/NEJMc2005073.

9. Naicker S, Yang CW, Hwang SJ, Liu BC, Chen JH, Jha V. The novel coronavirus 2019 epidemic and kidneys. Kidney Int. 2020 Mar 7. doi: 10.1016/j.kint.2020.03.001.

10. Wang D, Hu B, Hu C, Zhu F, Liu X, Zhang J, et al. Clinical characteristics of 138 hospitalized patients with 2019 novel coronavirus-infected pneumonia in Wuhan, China. JAMA. 2020 Feb 7. doi: 10.1001/jama.2020.1585

11. Li Z, Wu M, Guo J, Yao J, Liao X, Song S, et al. Caution on Kidney Dysfunctions of 2019-nCoV Patients. MedRvix. 2020. doi: 10.1101/2020.02.08.20021212

12. Cheng Y, Luo R, Wang K, Zhang M, Wang Z, Dong L, et al. Kidney impairment is associated with in-hospital death ofCOVID-19 patients. medRxiv. 2020. doi: $10.1101 / 2020.02 .18 .20023242$
13. Li W, Moore MJ, Vasilieva N, Sui J, Wong SK, Berne MA, et al. Angiotensin-converting enzyme 2 is a functional receptor for the SARS coronavirus. Nature. 2003;426:450-4. doi: 10.1038/nature02145

14. Raj VS, Mou H, Smits SL, Dekkers DH, Müller MA, Dijkman R, et al. Dipeptidyl peptidase 4 is a functional receptor for the emerging human coronavirus-EMC. Nature. 2013;495:251-4. doi: 10.1038/nature12005

15. Guan WJ, Ni ZY, Hu Y, Liang WH, Ou CQ, He JX, et al. Clinical characteristics of coronavirus disease 2019 in China. N Engl J Med. 2020. doi: 10.1056/NEJMoa2002032

16. Pan XW, Xu D, Zhang H, Zhou W, Wang LH, Cui XG Identification of a potential mechanism of acute kidney injury during the COVID-19 outbreak: a study based on single-cell transcriptome analysis. Intensive Care Med. 2020 Mar 31. doi: 10.1007/s00134-020-06026-1.

17. Sanders JM, Monogue ML, Jodlowski TZ, Cutrell JB. Pharmacologic treatments for coronavirus disease 2019 (COVID-19): a review. JAMA. 2020 Apr 13. doi: 10.1001/ jama.2020.6019.

18. Russell CD, Millar JE, Baillie JK . Clinical evidence does not support corticosteroid treatment for 2019-nCoV lung injury. Lancet. 2020;395:473-475. doi: 10.1016/S01406736(20)30317-2.

19. Ye M, Fu D, Ren Y, Wang F, Wang D, Zhang F, et al. Treatment with convalescent plasma for COVID-19 patients in Wuhan, China. J Med Virol. 2020. doi: 10.1002/ jmv.25882.

20. Ma Y, Diao B, Lv X, Zhu J, Liang W, Liu L, et al. 2019 novel coronavirus disease in hemodialysis (HD) patients: Report from one HD center in Wuhan, China. medRxiv. 2020 Jan 1. doi: 10.1101/2020.02.24.20027201.

21. Yang Y, Shi J, Ge S, Guo S, Xing X, Wang Y, et al. Effect of continuous renal replacement therapy on all-cause mortality in COVID-19 patients undergoing invasive mechanical ventilation: a retrospective cohort study. medRxiv. 2020: doi: 10.1101/2020.03.16.20036780.

Copyright $\odot 2020$ The Author(s); Published by Nickan Research Institute. This is an open-access article distributed under the terms of the Creative Commons Attribution License (http://creativecommons.org/licenses/by/4.0), which permits unrestricted use, distribution, and reproduction in any medium, provided the original work is properly cited. 